\title{
Incidencia del período vacacional de Navidad en el estado ponderal de escolares de Primaria
}

\author{
Melchor Martínez-Redondo ${ }^{a, *}$, Pedro Ángel Latorre-Román ${ }^{a}$ \\ $\mathbf{a}_{\text {Universidad de Jaén, España. }}$ \\ *melchor_mr@hotmail.com
}

Recibido el 5 de febrero de 2017; aceptado el 23 de julio de 2017; publicado el 29 de septiembre de 2017.

Incidencia del período vacacional de Navidad en el estado ponderal de escolares de Primaria

PALABRAS CLAVE
Índice de Masa
Corporal;
Niño;
Instituciones
Académicas;
Vacaciones y Feriados.

\section{RESUMEN}

Introducción: El objetivo de este estudio fue conocer si existen cambios en el índice de masa corporal (IMC) en el alumnado de Educación Primaria tras el período no escolar de vacaciones de Navidad y las diferencias entre niños con normopeso, sobrepeso y obesidad.

Material y Métodos: Estudio descriptivo Iongitudinal y prospectivo. Participaron 156 alumnos del colegio público Doctor Fleming de Jódar, Jaén (España), de 8 a 12 años de edad: 119 con normopeso, 18 con sobrepeso y 19 con obesidad. Se realizaron mediciones de talla, peso y cálculo del IMC en tres momentos: en período lectivo previo a las vacaciones de Navidad, tras período no lectivo vacacional (Navidad) y medición tras 20 días después de Navidad, con un intervalo de tiempo entre mediciones de 20 días.

Resultados: Los datos obtenidos (media [desviación estándar]) mostraron un aumento del IMC tras el período de Navidad en todos los grupos: normopeso: 0,09(0,43) kg/m²; sobrepeso: $0,29(0,46) \mathrm{kg} / \mathrm{m}^{2} ;$ y obesidad: $0,50(0,70) \mathrm{kg} / \mathrm{m}^{2}$ con diferencias significativas entre los de normopeso y obesidad ( $p=0,007)$. Transcurridos 20 días tras la Navidad, los niños con normopeso se aproximaron a los valores cercanos a la primera medición con diferencias significativas $(p<0,05)$ en relación con los niños con obesidad que son los que menos disminuyen su IMC $\left(0,04[0,56] \mathrm{kg} / \mathrm{m}^{2}\right.$ frente a $\left.0,38[0,50] \mathrm{kg} / \mathrm{m}^{2}\right)$.

Conclusiones: Los niños con obesidad incrementan en mayor medida su IMC tras el período de vacaciones de Navidad y no recuperan los valores iniciales en relación con los niños con normopeso. 
Incidence of the Christmas holiday period on the weight condition of Primary-school students

\section{KEYWORDS}

Body Mass Index;

Child;

Schools;

Holidays.

\section{CITA}

\section{ABSTRACT}

Introduction: The aim of this study was to analyze changes in body mass index (BMI) of primaryschool students and the differences between children with normal-weight, overweight and obesity after the Christmas holiday period.

Material and Methods: Longitudinal and prospective descriptive study was performed. A total of 156 students of the public school Doctor Fleming from Jódar, Jaén (Spain) participated in this study, aged 8 to 12 years: 119 with normal-weight, 18 with overweight and 19 with obesity. Measurements of height, weight, and BMI were taken at three moments: in the school period prior to the Christmas holiday, after a non-school period (Christmas holiday) and 20 days after the Christmas holiday, with an interval time between measurements of 20 days.

Results: The data (mean [standard deviation]) showed an increase in the BMI after the Christmas holiday period in all groups: normal-weight: $0.09(0.43) \mathrm{kg} / \mathrm{m}^{2}$; overweight: $0.29(0.46) \mathrm{kg} / \mathrm{m}^{2}$; and obesity: $0.50(0.70) \mathrm{kg} / \mathrm{m}^{2}$ with significant differences between normal-weight and obesity $(p=0.007)$. After 20 days after Christmas, children with normal-weight were approximated to near values to the first measurement with significant differences $(p<0.05)$ in relation to children whit obesity who were the ones that least decrease their BMI $\left(0.04[0.56] \mathrm{kg} / \mathrm{m}^{2}\right.$ versus $\left.0.38[0.50] \mathrm{kg} / \mathrm{m}^{2}\right)$.

Conclusions: Children with obesity showed higher values of BMI than normal-weight children during the non-school period, and they do not recover initial values in relation to normal-weight children.

\section{INTRODUCCIÓN}

La Organización Mundial de la Salud (OMS) considera la obesidad como una epidemia de distribución mundial ${ }^{1}$. La prevalencia de sobrepeso y obesidad ha sufrido un incremento en las últimas décadas llegando a límites alarmantes. Si las tendencias continúan, la probabilidad de alcanzar el objetivo de detener en el 2025 el aumento de la obesidad es prácticamente nula. La prevalencia de obesidad global alcanzará el $18 \%$ en hombres y superará el $21 \%$ en mujeres, y la obesidad severa superará el $6 \%$ en hombres y $9 \%$ en mujeres ${ }^{2}$. En España, el sobrepeso y obesidad son un importante problema de salud pública. En el grupo de edad de 8 a 17 años, en 2012, la prevalencia de sobrepeso era del $26 \%$ y la de obesidad del $12,6 \%$, por tanto, 4 de cada 10 jóvenes sufren exceso de peso ${ }^{3}$. Se ha comprobado que el excesivo incremento de peso en el niño es un riesgo para el aumento de la obesidad en la futura madurez ${ }^{4}$, ya que la probabilidad de que un individuo obeso de 6 años llegue a la edad adulta con obesidad supera el $50 \%$,5.6. A su vez, el niño activo físicamente tiene una mayor probabilidad de serlo en la madurez, manteniendo su actividad física (AF) durante mayor tiempo 7 .

La AF es útil en el control y prevención del sobrepeso y la obesidad $^{8,9}$. La inactividad física durante los primeros años de vida está reconocida actualmente como un importante factor coadyuvante en el incremento de los niveles de obesidad y de otros trastornos médicos graves que se observan en niños y adolescentes de Europa y de otros lugares ${ }^{10,11}$. Programas escolares basados en fomentar estilos de vida saludables, promocionando una alimentación balanceada y un incremento en la $A F$, han logrado influenciar positivamente en el índice de masa corporal (IMC) en niños de 6 a 16 años, reduciendo la prevalencia de sobrepeso y obesidad ${ }^{12}$. 
Los centros escolares constituyen un entorno adecuado para la promoción de la AF y la salud, ya que los escolares pasan gran parte del día en él. La clase de educación física y el recreo ofrecen las principales oportunidades de realizar AF dentro del colegio ${ }^{13,14}$.

Diversos estudios han analizado la influencia del período lectivo y no lectivo de vacaciones y su incidencia en el sobrepeso y obesidad en los niños, comprobándose que el período no lectivo de vacaciones es un momento de riesgo importante para el incremento del porcentaje de grasa corporal y del peso corporal, con mayor relevancia en niños con sobrepeso y obesidad; mientras que el período lectivo escolar produce una desaceleración del incremento de grasa y peso corporal ${ }^{15-19}$. Sin embargo, las referencias internacionales en cuanto al estado ponderal de población escolar se han basado, en la mayor parte de los casos, en el empleo del IMC como parámetro más operativo y preciso ${ }^{15,16,19}$.

A partir de las consideraciones anteriores, el objetivo de este estudio fue conocer la variabilidad del IMC de los niños de 8 a 12 años en dos períodos diferentes: el período escolar y el período no escolar de las vacaciones de Navidad, en función de las diferencias existentes entre niños con normopeso, sobrepeso y obesidad.

\section{MATERIAL Y MÉTODOS}

Estudio descriptivo longitudinal, realizado con una metodología cuantitativa y con recogida de datos de forma prospectiva. Participaron un total de 156 niños (68 niñas y 88 niños) de edades comprendidas entre 8 a 12 años del Colegio de Educación Infantil y Primaria Doctor Fleming de la localidad de Jódar, Jaén (España). La muestra tuvo un carácter de conveniencia y fue no probabilística. Como criterios de inclusión se tuvo en cuenta el no padecer discapacidad física, enfermedad metabólica como diabetes o enfermedad del tiroides, debido al posible efecto de interacción de medicamentos en las ganancias o pérdidas de peso ${ }^{20,21}$. Los padres firmaron un consentimiento informado de participación voluntaria de los niños en esta investigación e informaron sobre los elementos requeridos para la exclusión de los participantes. El estudio se realizó en cumplimento de las normas de la Declaración de Helsinki (versión 2013) y siguiendo las directrices de la Unión Europea en materia de Buenas Prácticas Clínicas (111/3976/88 de julio de 1990).

Las variables antropométricas recogidas fueron altura, peso e IMC. La altura (m), medida con un tallímetro (Seca 222, Hamburgo, Alemania) con el valor más cercano a 0,5cm. El peso (kg) se registró con una báscula (Seca 634, Hamburgo,
Alemania), con el valor más cercano a 0,1kg. EI IMC se obtuvo a partir de la fórmula: peso $(\mathrm{kg}) /$ altura $(\mathrm{m})^{2}$. Se tuvieron en cuenta los percentiles 85 y 95 de Sobradillo y Cols. $(2004)^{22}$ como punto de corte para clasificar a los niños/as con normopeso, sobrepeso y obesidad respectivamente en relación con el IMC, ya que éstos representan en mayor medida a la población española.

Tras la obtención de los permisos oportunos de los centros escolares y del consentimiento informado de los padres, se procedió en primer lugar a la obtención de los datos antropométricos de talla y peso de los niños, y la determinación del IMC corporal. Se realizaron 3 tomas de datos en diferentes momentos, con una separación entre tomas de 20 días. La primera toma se llevó a cabo justo el día previo al período no lectivo de Navidad, la segunda toma al día siguiente de haber finalizado el período no lectivo (20 días de vacaciones de Navidad), y la tercera toma tras 20 días (con respecto a la segunda toma). Todas las tomas de datos se realizaron en las instalaciones y aulas del colegio seleccionado, en la hora previa al recreo escolar (11:00-12:00 horas), sin zapatos y mínima ropa (sin abrigo o jersey), y antes de tomar el desayuno.

Los datos de este estudio se han hallado mediante el programa estadístico SPSS, v.19.0 para Windows, (SPSS Inc, Chicago, EE. UU.). El nivel de significación se fijó en $p \leq 0,05$. Las variables fueron descritas con media y desviación típica para variables continuas y porcentajes para variables cualitativas. Se comprobó la distribución normal de los datos y la igualdad de varianzas mediante pruebas de Kolmogorov-Smirnov y contraste de Levene respectivamente. Las diferencias entre grupos se analizaron mediante análisis de varianza con medidas repetidas (ANOVA $3 \times 3$ ) realizando pruebas post-hoc (Bonferroni). El tamaño del efecto se determino mediante Eta ${ }^{2}$ parcial.

\section{RESULTADOS}

Participaron un total de 156 niños de edades comprendidas entre 8 a 12 años (edad = 9,66 [1,20] años), 88 niños $(56,41 \%)$ y 68 niñas $(43,59 \%)$, de los cuales 119 niños con normopeso, 18 niños con sobrepeso y 19 niños con obesidad.

En la Tabla 1 se exponen los resultados antropométricos (peso, talla e IMC) de las tres tomas de datos realizadas en los diferentes grupos. En la toma 1 los niños del grupo norpomeso mostraron un IMC de $17,34(2,39) \mathrm{kg} / \mathrm{m}^{2}$, los del grupo sobrepeso $22,21(1,84) \mathrm{kg} / \mathrm{m}^{2}$ y los del grupo obesidad $26,33(1,96) \mathrm{kg} / \mathrm{m}^{2}$. En la toma 2 todos los grupos presen- 
Tabla 1. Peso, Talla e IMC en niños con normopeso, niños con sobrepeso y niños con obesidad.

\begin{tabular}{|c|c|c|c|c|c|c|}
\hline Variables & Grupos & $\begin{array}{c}\text { T1 } \\
\text { Media (DE) }\end{array}$ & $\begin{array}{c}\text { T2 } \\
\text { Media (DE) }\end{array}$ & $\begin{array}{c}\text { T3 } \\
\text { Media (DE) }\end{array}$ & $\begin{array}{c}p \text {-valor } \\
\text { (grupo x tiempo) }\end{array}$ & $\begin{array}{c}\text { Eta }^{2} \\
\text { parcial }\end{array}$ \\
\hline $\begin{array}{l}\text { Peso (kg) } \\
\text { p-valor (tiempo x grupo) } \\
\text { Eta }^{2} \text { parcial }\end{array}$ & $\begin{array}{l}\text { G1 } \\
\text { G2 } \\
\text { G3 }\end{array}$ & $\begin{array}{c}33,80(7,61)_{a} \\
43,68(7,21)_{a} \\
56,19(7,48)_{a} \\
<0,001 \\
0,496\end{array}$ & $\begin{array}{c}34,02(7,58)_{b} \\
44,29(7,64)_{b} \\
57,11(7,32)_{b} \\
<0,001 \\
0,511\end{array}$ & $\begin{array}{c}33,96(7,63)_{a b} \\
44,04(7,67)_{a b} \\
57,04(7,31)_{b} \\
<0,001 \\
0,507\end{array}$ & $\begin{array}{c}0,001 \\
0,001 \\
<0,001\end{array}$ & $\begin{array}{l}0,083 \\
0,093 \\
0,190\end{array}$ \\
\hline $\begin{array}{l}\text { Talla (m) } \\
\text { p-valor (tiempo x grupo) } \\
\text { Eta }^{2} \text { parcial }\end{array}$ & $\begin{array}{l}\text { G1 } \\
\text { G2 } \\
\text { G3 }\end{array}$ & $\begin{array}{c}1,38(0,08)_{a} \\
1,39(0,06) \\
1,45(0,07)_{a} \\
0,005 \\
0,067\end{array}$ & $\begin{array}{c}1,39(0,08)_{b} \\
1,39(0,06) \\
1,46(0,07)_{a} \\
0,005 \\
0,068\end{array}$ & $\begin{array}{c}1,39(0,08)_{c} \\
1,39(0,07) \\
1,46(0,07)_{b} \\
0,005 \\
0,067\end{array}$ & $\begin{array}{c}<0,001 \\
0,125 \\
0,008\end{array}$ & $\begin{array}{l}0,266 \\
0,027 \\
0,062\end{array}$ \\
\hline $\begin{array}{l}\text { IMC }\left(\mathrm{kg} / \mathrm{m}^{2}\right) \\
\text { p-valor (tiempo x grupo) } \\
\text { Eta }^{2} \text { parcial }\end{array}$ & $\begin{array}{l}\text { G1 } \\
\text { G2 } \\
\text { G3 }\end{array}$ & $\begin{array}{c}17,34(2,39)_{a} \\
22,21(1,84)_{a} \\
26,33(1,96)_{a} \\
<0,001 \\
0,646\end{array}$ & $\begin{array}{c}17,42(2,42)_{b} \\
22,49(2,06)_{b} \\
26,72(1,89)_{b} \\
<0,001 \\
0,654\end{array}$ & $\begin{array}{c}17,35(2,42)_{a} \\
22,32(2,06)_{a b} \\
26,64(2,06)_{b} \\
<0,001 \\
0,651\end{array}$ & $\begin{array}{c}0,002 \\
0,001 \\
<0,001\end{array}$ & $\begin{array}{l}0,076 \\
0,087 \\
0,137\end{array}$ \\
\hline
\end{tabular}

T1: Analizado en período lectivo antes de las vacaciones de Navidad; T2: Analizado en el período de vacaciones de Navidad; T3: Analizado tras 20 días del período de Navidad; G1: Grupo Normopeso;

G2: Grupo Sobrepeso. G3: Grupo Obesidad; DE: desviación estándar. IMC: índice de masa corporal; $a, b, c:$ diferente letra subíndice indica diferencias significativas $(p<0,05)$ en las variables grupo $x$ tiempo.

taron un aumento significativo $(p<0,05)$ en los valores del IMC (grupo normopeso: $+0,09[0,43] \mathrm{kg} / \mathrm{m}^{2}$; grupo sobrepeso: $+0,29[0,46] \mathrm{kg} / \mathrm{m}^{2}$; y grupo obesidad: $+0,50[0,70] \mathrm{kg} /$ $\left.\mathrm{m}^{2}\right)$ con respecto a la toma 1 . Además, existieron diferencias significativas $(p<0,05)$ entre el grupo con normopeso y obesidad, este último experimentó los mayores incrementos en el IMC (Figura 1). En la toma 3, se observó una disminución en todos los grupos respecto a la toma 2 (grupo normopeso:

Figura 1. Incremento del IMC (kg/m²): toma 2 (T2) menos toma 1 (T1), en niños con normopeso, niños con sobrepeso y niños con obesidad.

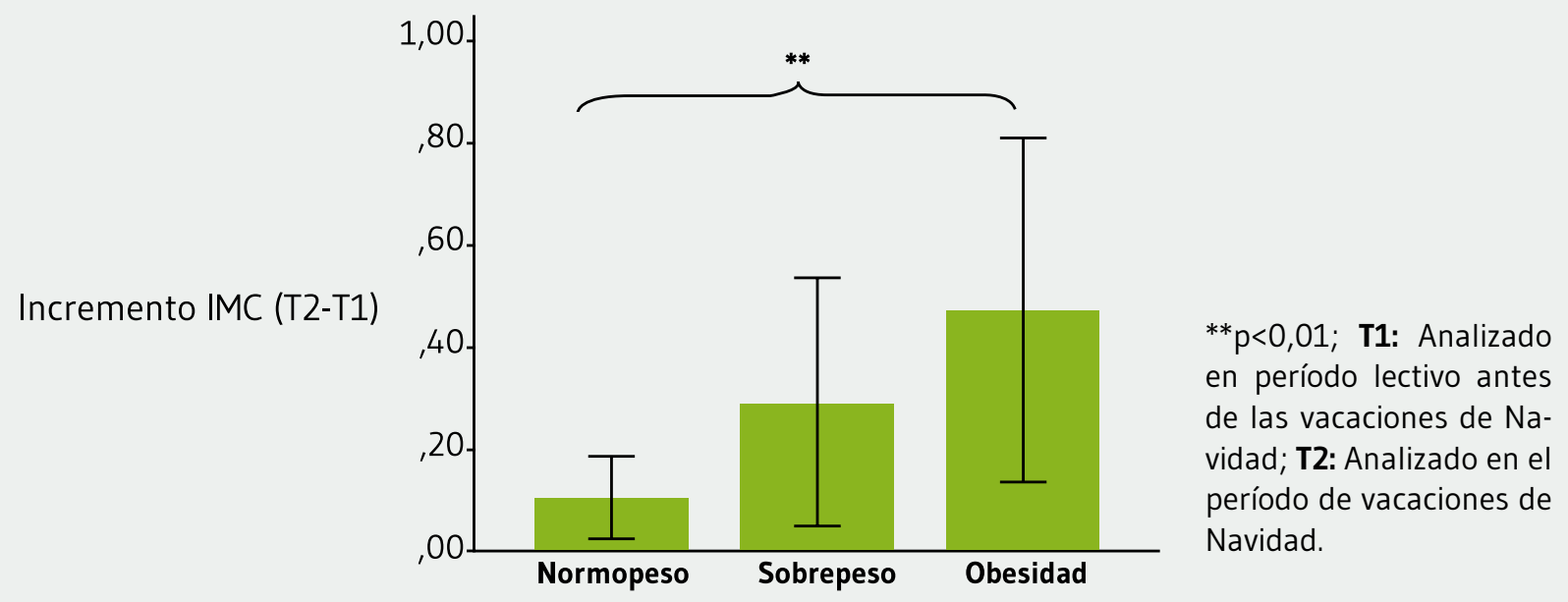


$-0,07[0,51] \mathrm{kg} / \mathrm{m}^{2} ;$ grupo sobrepeso: $-0,17[0,42] \mathrm{kg} / \mathrm{m}^{2} ; \mathrm{y}$ grupo obesidad: $\left.-0,08[0,26] \mathrm{kg} / \mathrm{m}^{2}\right)$. Comparando la toma 1 con la toma 3 se observó un aumento del IMC en todos los grupos (grupo normopeso: $+0,04[0,56] \mathrm{kg} / \mathrm{m}^{2}$; grupo sobrepeso: $+0,11[0,60] \mathrm{kg} / \mathrm{m}^{2}$; y grupo obesidad: $\left.+0,38[0,50] \mathrm{kg} / \mathrm{m}^{2}\right)$, mientras que el grupo de obesidad se situó de forma significativa $(p<0,05)$ con valores por encima de la toma 1 . En la Figura 2 se puede observar que entre el grupo de normopeso y obesidad existieron diferencias significativas $(p<0,05)$ en el nivel de reducción de los valores de IMC (toma 3 - toma 1 ).

\section{Discusión}

El hallazgo más importante de este estudio muestra un aumento del IMC de los niños tras un período de 20 días no lectivos, con diferencias significativas entre el grupo de normopeso y obesidad, siendo este último el que mayor incremento experimenta. A la vuelta al período escolar, se observa una disminución en los valores del IMC en los diferentes grupos, y los niños con normopeso se aproximan a los valores cercanos a la primera toma, mientras que los niños con obesidad es el grupo que menos disminuye su IMC y más se aleja de su IMC inicial.

Este estudio corrobora en primer lugar los datos obtenidos en otros estudios que han analizado en niños otro período vacacional diferente más largo, como es el de verano. Los resultados expuestos anteriormente están en consonancia con los obtenidos por Plaza y Cols. en $2008^{15}$, Von Hippel y Workman en $2016^{19}$ y Martínez-López y Cols. en 20117. En el estudio de Plaza y Cols. ${ }^{15}$ la prevalencia de obesidad en la primera evaluación fue de $23,5 \%$ y la de sobrepeso del $33,3 \%$, en la segunda medición los valores ascendieron a 28,2 y $36,4 \%$ respectivamente. Comparando el valor medio del IMC antes y después del verano, se observó una diferencia significativa, siendo superior después del verano (18,37 frente a $\left.18,73 \mathrm{~kg} / \mathrm{m}^{2}\right)$. Por otro lado, Von Hippel y Workman ${ }^{19}$, observaron que al finalizar el período de vacaciones estivales la prevalencia de sobrepeso pasó de un $23,3 \%$ a un $28,7 \%$, mientras que la de obesidad se le elevó de un $8,9 \%$ a un $11,5 \%$. Martínez-López y Cols. ${ }^{17}$, empleando la bioimpedancia eléctrica obtuvieron un aumento acumulado de grasa corporal de los escolares de 1,38\% durante el período vacacional de verano.

Por consiguiente, los resultados de este estudio ponen de relieve que una vez que se vuelve al período lectivo el IMC de los niños disminuye, sobretodo en el grupo de normopeso y con diferencias significativas con el grupo de obesos, lo cual coincide con los datos obtenidos por Von Hippel y Workman. en $2016^{19}$, según los cuales la prevalencia de obesidad se redujo ligeramente durante la época de clases (aunque no se modificó la prevalencia de sobrepeso).

En comparación con el estudio de Martínez-López y Cols. en $2011^{17}$, en relación al análisis realizado en los grupos de normopeso y sobrepeso, los sujetos con sobrepeso y características endomórficas regulan mejor su cantidad de grasa corporal durante el período no lectivo que los adolescentes con normopeso. Los datos obtenidos del presente estudio son divergentes, ya que los niños con sobrepeso y obesidad

Figura 2. Incremento del IMC ( $\left.\mathrm{kg} / \mathrm{m}^{2}\right)$ : toma 3 (T3) menos toma 1 (T1), en niños con normopeso, niños con sobrepeso y niños con obesidad

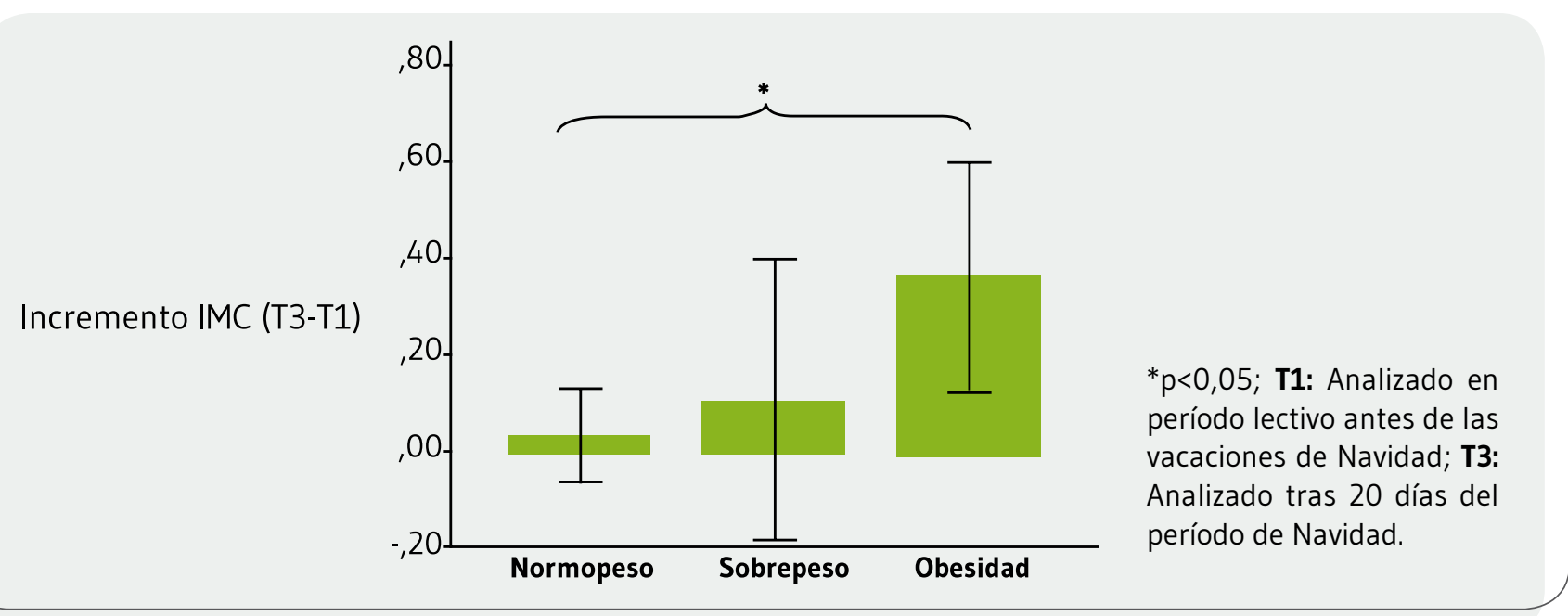


son los que aumentan en mayor medida su IMC durante el período no lectivo, regulándose mejor los de normopeso. Estas diferencias en los resultados se pueden atribuir a las diferencias de las edades de los niños, en el estudio realizado por Martínez-López y Cols. ${ }^{17}$ los participantes eran adolescentes de Educación Secundaria (edad = 13,89 [0,80] años) y la adolescencia es un período donde se producen intensos cambios físicos: hormonales, de crecimiento y composición corporal23.

El estudio realizado en Chile por Cristi-Montero y Cols. en $2014^{18}$, que valoró la variación de la composición corporal durante las vacaciones de invierno (16 días, julio) y las vacaciones nacionales (9 días, septiembre) en niños, puso de manifiesto un aumento significativo en el peso $1+600 \mathrm{~g}$ $y+510 g$ durante vacaciones nacionales y de invierno, respectivamente) y el porcentaje de masa corporal (+0,51\%) en ambos períodos, por lo que guardan relación con los datos obtenidos en el presente estudio.

De manera más específica con este estudio, una investigación realizada en el período vacacional de Navidad en niños ${ }^{16}$ señala que los niños con normopeso aumentaron su IMC en $+0,18 \mathrm{~kg} / \mathrm{m}^{2}$ y los niños con sobrepeso/obesidad en $+0,37 \mathrm{~kg} / \mathrm{m}^{2}$. Estos datos guardan relación con los obtenidos en el presente estudio (IMC: grupo normopeso = $0,09[0,43] \mathrm{kg} / \mathrm{m}^{2}$; grupo sobrepeso $=0,29[0,46] \mathrm{kg} / \mathrm{m}^{2} ; \mathrm{y}$ grupo obesidad $\left.=0,50[0,70] \mathrm{kg} / \mathrm{m}^{2}\right)$ y ponen de manifiesto que la Navidad es un período crítico para el incremento de peso en niños con sobrepeso y obesidad.

Por tanto, en este estudio se destaca que durante el período lectivo los niños presentan un mayor control de su estado ponderal. El establecimiento de rutinas como por ejemplo levantarse para ir a clase, el desplazamiento activo a los centros educativos ${ }^{24,25}$, el realizar dos horas de práctica de AF (educación física) a la semana, los períodos de recreo donde se favorece el movimiento del alumnado $0^{14,26}$ o la participación en actividades extraescolares ${ }^{27}$ como los programas institucionalizados "Escuelas Deportivas" de las comunidades autónomas ( 2 horas semanales) son factores que inciden directamente sobre el control del IMC del alumnado.

Como limitaciones del estudio es necesario tener presente que estas mediciones se han realizado sobre el período vacacional de Navidad, fechas en las que los niños realizan una menor $\mathrm{AF}^{18}$, y tienen un aumento del tiempo de sueño ${ }^{18,28}$. Otros períodos no lectivos deberían ser analizados. Además, no se han analizado las diferencias entre sexos, lo que supone otra limitación a tener en cuenta. También se han utilizado escasos parámetros antropométricos, se podría haber incluido otros como la circunferencia de la cintura o el somatotipo. Por último, los datos de este estudio se refieren a una muestra por conveniencia por lo que los resultados deben ser tomados con cautela. Además, la monitorización precisa de la AF durante los períodos lectivos y no lectivos analizados, nos aportaría información más exacta para interpretar los resultados. Finalmente como fortaleza del estudio destacamos el preciso seguimiento de la muestra seleccionada durante el intervalo de tiempo previsto.

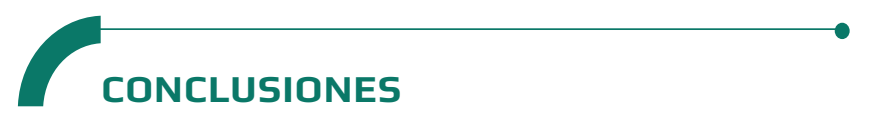

Los niños con obesidad incrementan en mayor medida su IMC tras el período de vacaciones de Navidad y no recuperan los valores iniciales en relación con los niños con normopeso. Las vacaciones de Navidad, por lo tanto, son un período de riesgo en escolares ya que contribuyen a un aumento del IMC, afectando principalmente a los niños obesos. Son necesarios más estudios para corroborar estos datos.

\section{CONFlicto de intereses}

Los autores expresan que no hay conflictos de interés al redactar el manuscrito.

\section{REFERENCIAS}

(1) Organización Mundial de la Salud. Plan de Acción Mundial para la Prevención y el Control de las Enfermedades No Transmisibles 2013-2020: Proyecto Revisado y Actualizado [Internet]. Ginebra: OMS; 2013. Disponible en: http://www. who.int/cardiovascular_diseases/15032013_updated revised_draft_action_plan_spanish.pdf

(2) NCD Risk Factor Collaboration (NCD-RisC). Trends in adult body-mass index in 200 countries from 1975 to 2014: a pooled analysis of 1698 population-based measurement studies with $19 \cdot 2$ million participants. Lancet. 2016; 387(10026): 1377-96.

(3) Sánchez-Cruz J, Jiménez-Moleón J], Fernández-Quesada F, Sánchez MJ. Prevalencia de obesidad infantil y juvenil en España en 2012. Rev Esp Cardiol. 2013; 66(5): 371-6.

(4) Whitaker RC, Wright JA, Pepe MS, Seidel KD, Dietz WH. Predicting obesity in young adulthood from childhood and parental obesity. N Engl J Med. 1997; 337(13): 869-73.

(5) Downey DB, von Hippel PT, Broh BA. Are Schools the Great Equalizer? Cognitive Inequality during the Summer Months and the School Year. Am Sociol Rev. 2004; 69(5): 613-35.

(6) Chan SSC, Lam TH, Salili F, Leung GM, Wong DCN, Botelho $\mathrm{RJ}$, et al. A randomized controlled trial of an individualized motivational intervention on smoking cessation for parents of sick children: a pilot study. Appl Nurs Res. 2005; 18(3): 178-81. 
(7) Malina RM. Tracking of physical activity and physical fitness across the lifespan. Res Q Exerc Sport. 1996; 67(3 Suppl): S48-57.

(8) Gupta N, Goel K, Shah P, Misra A. Childhood obesity in developing countries: epidemiology, determinants, and prevention. Endocr Rev. 2012; 33(1): 48-70.

(9) Hills AP, Andersen LB, Byrne NM. Physical activity and obesity in children. Br J Sports Med. 2011; 45(11): 866-70.

(10) Livingstone MB. Childhood obesity in Europe: a growing concern. Public Health Nutr. 2001; 4(1A): 109-16.

(11) Speiser PW, RudolfMCJ, Anhalt H, Camacho-Hubner C, Chiarelli F, Eliakim A, et al. Childhood obesity. J Clin Endocrinol Metab. 2005; 90(3): 1871-87.

(12) Pérez L, Raigada J, Collins A, Mauricio S, Felices A, Jiménez $S$, et al. Efectividad de un programa educativo en estilos de vida saludables sobre la reducción de sobrepeso y obesidad en el Colegio Robert M. Smith; Huaraz, Ancash, Perú. Acta Méd Peruana. 2008; 25(4): 204-9.

(13) Naylor P-J, McKay HA. Prevention in the first place: schools a setting for action on physical inactivity. Br ] Sports Med. 2009; 43(1): 10-3.

(14) Beighle A, Morgan CF, Le Masurier G, Pangrazi RP. Children's physical activity during recess and outside of school. J Sch Health. 2006; 76(10): 516-20.

(15) Plaza J, Siurana JM, Vergara L, Mateos F, Romero MI. Prevalencia de Obesidad en escolares. Rev Clin Med Fam. 2008; 2(3): 106-10.

(16) Branscum P, Kaye G, Succop P, Sharma M. An Evaluation of Holiday Weight Gain Among Elementary-aged Children. J Clin Med Res. 2010; 2(4): 167-71.

(17) Martínez-López EJ, Redecillas MT, Moral JE. Grasa corporal mediante bioimpedancia eléctrica en periodo escolar y no escolar. Rev Int Med Cienc Act Fís Deporte. 2011; 10(41): 77-94.

(18) Cristi-Montero C, Bresciani G, Alvarez A, Arriagada V, Beneventi A, Canepa V, et al. Critical periods in the variation in body composition in school children. Nutr Hosp. 2014; 30(4): 782-6.
(19) von Hippel PT, Workman J. From Kindergarten Through Second Grade, U.S. Children's Obesity Prevalence Grows Only During Summer Vacations. Obesity (Silver Spring). 2016; 24(11): 2296-300.

(20) Rogero ME, Albañil MR, Sánchez M, Rabanal A, Olivas A, García C. Prevalencia de resistencia a insulina en una población de jóvenes adultos. Relación con el estado ponderal. Endocrinol Nutr. 2012; 59(2): 98-104.

(21) Mardones F, Arnaiz P, Barja S, Giadach C, Villarroel L, Domínguez $A$, et al. Estado nutricional, síndrome metabólico y resistencia a la insulina en niños de Santiago, Chile. Nutr Hosp. 2013; 28(6): 1999-2005.

(22) Sobradillo B, Aguirre A, Aresti U, Bilbao A, Fernández-Ramos C, Lizárraga A, et al. Curvas y tablas de crecimiento (Estudio longitudinal y transversal). Bilbao: Fundación Faustino Orbegozo Eizaguirre; 2004.

(23) Iglesias JL. Desarrollo del adolescente: aspectos físicos, psicológicos y sociales. Pediatr Integral. 2013; 17(2): 88-93.

(24) Tudor-Locke C, Ainsworth BE, Adair LS, Popkin BM. Objective physical activity of filipino youth stratified for commuting mode to school. Med Sci Sports Exerc. 2003; 35(3): 465-71.

(25) Davison KK, Werder JL, Lawson CT. Children's active commuting to school: current knowledge and future directions. Prev Chronic Dis. 2008; 5(3): A100.

(26) Escalante Y, Backx K, Saavedra JM, García-Hermoso A, Domínguez AM. Relationship between daily physical activity, recess physical activity, age and sex in scholar of primary school, Spain. Rev Esp Salud Publica. 2011; 85(5): 481-9.

(27) Busto R, Amigo I, Fernández C, Herrero J. Actividades extraescolares, ocio sedentario y horas de sueño como determinantes del sobrepeso infantil. Int J Psych Psychol Ther. 2009; 9(1): 59-66.

(28) Wing YK, Li SX, Li AM, Zhang J, Kong APS. The effect of weekend and holiday sleep compensation on childhood overweight and obesity. Pediatrics. 2009; 124(5): e994-1000. 